\begin{tabular}{|l|c|c|c|c|c|}
\hline Revista Clío América & ISSN: 1909-941X & Vol. 10 & No. 20 & Julio - Diciembre de 2016 & 171 - 185 \\
\hline \multicolumn{6}{|c|}{ DOI: http://dx.doi.org/10.21676/23897848.1875 } \\
\hline
\end{tabular}

\title{
La política de vivienda y la adjudicación de créditos hipotecarios en la ciudad de Cúcuta
}

\author{
Housing policy and the granting of mortgage loans in the city of Cúcuta
}

Jhon Antuny Pabón-León

Magister en Gerencia de Empresas mención

Finanzas, Docente de la Universidad Francisco de Paula Santander, Cúcuta, Colombia.

Email: jhonantuny@ufps.edu.co

Tipología:

Artículo de Reflexión

Fecha de Recibido:

Abril 29 de 2016

Fecha de Aceptación:

Agosto 29 de 2016

Para citar este artículo:

Pabón, L. J. (2016). La política de vivienda y la adjudicación de créditos hipotecarios

en la ciudad de Cúcuta.

Clío América, 10 (20), pp. 171 - 185
Resumen: Este artículo de reflexión se enfoca en la política de vivienda y la adjudicación de créditos hipotecarios en la ciudad de Cúcuta a partir de la promulgación de la política de vivienda. Es un estudio descriptivo de enfoque cuantitativo, no experimental, transeccional apoyado en revisión documental atendiendo tres ejes temáticos: las políticas de vivienda del gobierno, el mercado de la vivienda y el financiamiento de vivienda. La vivienda es hoy en día uno de los problemas que más afecta a la sociedad actual debida, entre otros factores, al crecimiento de las ciudades y junto a ello las necesidades de tenencia y construcción de vivienda. La crisis de vivienda en Colombia ha sido uno de los problemas económicos más agobiantes en los últimos años y en consecuencia se diseñaron nuevos instrumentos para dar acceso efectivo a vivienda y atender a los potenciales beneficiarios. Surge así la inquietud por conocer cuál fue el impacto en la adjudicación de créditos hipotecarios en la ciudad de Cúcuta a partir de la ley promulgación de la política de vivienda. Se concluye que, aunque las opiniones difieren sobre la efectividad de la política de vivienda evidentemente el programa presentó un balance positivo en la ciudad de Cúcuta.

Palabras Clave: tipo de vivienda, políticas de vivienda, hipotecas, mercado de vivienda

JEL: D60, G21, I38, R21

Abstract: This reflection article focuses on housing policy and the granting of mortgage loans in the city of Cúcuta from promulgation of housing policy. It is a descriptive study of quantitative approach, non-experimental, transectional supported in document review addressing three thematic areas: housing policies of the Government, the housing market and housing finance. The House today on day one of the problems that most affect society due among other factors to the growth of cities and next to it is tenure and housing needs. The crisis of housing in Colombia has been one of the most crippling economic problems in recent years and as a result new instruments are designed to provide effective access to housing and care for the potential beneficiaries. Thus arises the concern about what was the impact on the allocation of mortgage loans in the city of Cúcuta from promulgation of housing policy? It is concluded that although opinions differ on the effectiveness of housing policy clearly program presented a positive balance in the city of Cúcuta.

Keywords: type of housing, housing, mortgages, housing market policies 


\section{Introducción}

La vivienda es considerada una de las necesidades más básicas de todo ser humano, de hecho, el derecho a la vivienda es un derecho universal reconocido para todas las personas en la Declaración Universal de los Derechos Humanos de 1948 y el Pacto Internacional de Derechos Económicos, Sociales y Culturales de 1966. En el Artículo 25 de la Declaración Universal de los Derechos Humanos dice: "toda persona tiene derecho a un nivel de vida adecuado que le asegure, así como a su familia, la salud y el bienestar y en especial la alimentación, el vestido, la vivienda, la asistencia médica y los servicios sociales necesarios". En razón de esto, para el Comité de Derechos Económicos, Sociales y Culturales de la ONU, el derecho a la vivienda no es el simple hecho de tener un tejado por encima de la cabeza o una comodidad. Es el derecho a vivir en seguridad, paz y dignidad en alguna parte.

Hoy en día se considera que la vivienda es uno de los problemas con más impacto a la familia acentuándose esto en los estratos menos favorecidos de la sociedad. Es un hecho que en el último siglo la población urbana superó a la población rural trayendo como consecuencia el crecimiento de las ciudades y junto a ello las necesidades de tenencia y construcción de vivienda.

Ahora bien, en Colombia de acuerdo con los cálculos del Ministerio de Vivienda citado por el Departamento Nacional de Planeación

...el $48,25 \%$ de los hogares que presentan algún tipo de carencia asociada al déficit cuantitativo de vivienda tienen ingresos por debajo de los 1,4 salarios mínimos mensuales legales vigentes y concentran buena parte de la población en condición de informalidad laboral y se supone que en este segmento no opera el modelo de cierre financiero. (2016, p.2)

Con base en las cifras presentadas es evidente que este segmento de la población requiere del apoyo directo del Estado a través de subsidios encaminados a coadyuvar en el acceso efectivo a la vivienda y a propiciar la generación de oferta donde sea escasa 0 inexistente.
Por su parte, Carrión (2005, p.1) señala que 1.500 millones de personas que viven en las ciudades pueden ser consideradas como población pobre, lo que representa más del $20 \%$ de la población mundial. Evidentemente esto trae un reto importante para atender las necesidades de vivienda aunado a los requerimientos en el desarrollo y ordenamiento urbano. Dentro de este marco se plantea que existe una gran desigualdad por un lado los que cuentan y viven con las condiciones que cubren sus necesidades básicas incluyendo vivienda y los que viven en las zonas periféricas donde se generaliza una exclusión social que alcanza a todos los niveles donde la vivienda ocupa factor preponderante.

A sabiendas de que se puede considerar utópico el planteamiento se supone que para hacer efectivo el derecho a la vivienda es necesario atacar las causas profundas del no acceso a la vivienda en el mundo. En atención a esta problemática Kothari (citado por Golay \& Özden) ha identificado entre dichas causas especialmente:

la especulación del terreno y la propiedad, las expropiaciones y los desalojos forzosos, el éxodo rural y el crecimiento de los barrios, la discriminación contra los grupos vulnerables incluidas las mujeres, los niños, los refugiados, los inmigrantes y las personas ancianas o con discapacidades, las catástrofes naturales y los conflictos armados. (2007, p.4)

En relación a este planteamiento, Golay \& Özden (2007, p.4) opinan que se requiere que el Estado juegue un papel más importante que conlleve a asegurar a los sectores más vulnerables el derecho al acceso a una vivienda digna como complemento de una mejor calidad de vida y garantice el respeto a sus derechos humanos y los defienda de atropellos tales como los desalojos forzosos. Aunque esta situación es ilegal es evidente que se observa en diferentes partes del mundo sin distingo de culturas o sistemas políticos y económicos. Nos preguntamos si entonces es un fenómeno sociológico o antropológico.

Por su parte Tella (s.f) en "La función social del suelo vs el derecho de propiedad" reseña que:

la política de vivienda en Europa se centra en nuevas dimensiones: la capacidad de elección, haciendo referencia 
tanto a la diversidad en el régimen de tenencia como a la cohesión social y a la variedad de clases sociales, edades y circunstancias en los enclaves de vivienda (en Francia, Finlandia, Irlanda o el Reino Unido); el derecho no sólo a la vivienda sino también a un entorno saludable, seguro y ecológicamente sostenible, a la ciudad, a un medio ambiente sano y a la calidad de vida (en Suecia, Francia, Irlanda o Finlandia); y el derecho a una vivienda con un precio razonable (Suecia o Finlandia). (p.1)

En razón de lo expuesto se puede afirmar que la problemática del tema de la vivienda y hábitat presenta ribetes generales y no solo se exteriotiza en países del tercer mundo. Evidentemente la problemática social generada por la necesidad de vivienda es un tema bastante sensible que además ha sido tomado como bandera política en algunos países.

Con carácter retrospectivo en el ámbito global se referencian tres investigaciones sobre el tema: En, Chile Zapata (2003) expuso que "las actuales tendencias de desarrollo urbano, potencialmente, podrían estar afectas a un nuevo proceso de exclusión social, que atañe tanto a los grupos sociales como a los territorios (...)" (p.13). Mientras que en España, Alcalá (2005) expuso: "en la actualidad están aumentando los problemas residenciales y la vivienda está actuando en muchos casos como un factor de deterioro y vulnerabilidad social y la política de vivienda surge como una respuesta pública a esta incapacidad" (p.81). Por su parte en Rodríguez (2010) se hace referencia al ejemplo español, donde se concluye que la garantía en el acceso a la vivienda sigue siendo una asignatura pendiente.

De hecho, en Colombia actualmente la oferta de vivienda se ajusta para familias con ingresos superiores a 1,5 salarios mínimos y no a la mayoría de los hogares colombianos. El resultado nacional del estudio de Déficit Habitacional muestra una consistente disminución del déficit de vivienda que estaría cercano al 16,4\% (1.647.093 unidades) de los hogares urbanos en 2012 frente al 27\% del Censo 2005, el dato más reciente sobre el tema, tal como lo indicó el Ministro de Vivienda Luis Felipe Henao en el artículo "Déficit de Vivienda Bajó en Colombia" (2014).
En atención a la problemática expuesta nace el interés por conocer cuál fue el impacto en la adjudicación de créditos hipotecarios en la ciudad de Cúcuta a partir de la ley 1537 de 2012, dado que desde el punto de vista social algunos análisis consideran que la vivienda es el segundo problema social más grande del país. El trabajo se corresponde con un estudio transeccional descriptivo de enfoque cuantitativo, no experimental, apoyado en una revisión documental atendiendo tres ejes temáticos: las políticas de vivienda del gobierno, el mercado de la vivienda y el financiamiento de vivienda. Con esto se pretende conocer el comportamiento de las variables en la ciudad de Cúcuta. El trabajo está organizado en cuatro partes: La primera incluye la introducción; en la parte dos se exponen conceptos y teorías sobre el tema de la vivienda además se expone el método de trabajo; en la parte tres se presenta el análisis de la información y resultados; la parte cuatro corresponde a la reflexión final, finalmente se listan las referencias bibliográficas que apoyan el estudio.

\section{Reseña histórica del sistema de financiamiento hipotecario en Colombia}

De acuerdo a González y García (citados por Cárdenas y Hernández, 2006, p.9) “(...) la evolución del sistema de financiamiento hipotecario en Colombia se puede dividir en dos grandes etapas: antes y después de la creación de la Unidad de Poder Adquisitivo Constante (UPAC) en 1972". Igualmente se encontró en Cárdenas y Hernández (2006) que:

(...) los orígenes del crédito hipotecario en Colombia se remontan a la creación del Banco Agrícola Hipotecario en 1924 y del Banco Central Hipotecario $(\mathrm{BCH})$ en 1932. Este último se convirtió en la principal (y en ocasiones única) fuente de crédito de largo plazo hasta la creación del sistema UPAC en los años setenta, como mecanismo de cuenta cuyo valor en moneda legal se ajustaba diariamente en función de la corrección monetaria. Así pues en 1972 se crearon las Corporaciones de Ahorro y Vivienda (CAV), pese a sus resultados, el sistema fue cuestionado a comienzos de los años ochenta debido a su escasa penetración en 
los estratos medios bajos y bajos. La Unidad de Poder Adquisitivo Constante (UPAC) fue ajustada diariamente por medio de la denominada corrección monetaria, desde su creación en septiembre de 1972 hasta su desaparición en diciembre de 1999. (p.9)

Igualmente, Cárdenas y Hernández (2006) señalan

La crisis del sector de financiamiento hipotecario es uno de los hechos más significativos en la evolución reciente de la economía colombiana, caracterizada por décadas de gran estabilidad en un ambiente de crecimiento continuo. En su opinión dado el deterioro de la calidad de la cartera hipotecaria a partir de junio de 1998, el gobierno decretó la emergencia económica en noviembre de 1998 que dispuso recursos a través del Fondo de Garantías de Instituciones Financieras (Fogafín) para otorgar créditos de alivio a los deudores hipotecarios con una tasa de interés subsidiada. (p.15)

Este análisis general representa el contexto de lo que representó la crisis económica y financiera en Colombia.

Posteriormente exponen Cardenas \& Hernandez (2006) "se introdujo a partir de enero de 2000 una nueva unidad de valor real (UVR) ajustada diariamente en función de la inflación del mes inmediatamente anterior, de hecho, los créditos denominados en UPAC se transformaron en créditos en UVR (p.20).

Sin embargo, esta no era la única causa de la crisis pues según Mejía, 2005 “(...) los bancos se mantenían renuentes a otorgar créditos hipotecarios como consecuencia de la alta morosidad de los deudores sucedida a finales de los noventa" (p.9). En el marco de las observaciones anteriores se comparte la opinión de Cárdenas y Hernández (2006) cuando afirman "la crisis hipotecaria que pudo haber sido considerada como un factor coyuntural de fines de los noventa se convirtió en un problema estructural que afectó al sector durante cinco años" (p.19).

\section{Programa de Vivienda de Interés Social en Colombia}

Henao (2013) señaló:

(...) en abril de 2011, el BID publicó los resultados de una evaluación del Programa de Vivienda de Interés Social en Colombia, la evaluación pretendía evaluar el acceso efectivo de la población objetivo al programa de vivienda de interés social en Colombia si como al mejoramiento de las condiciones de la vivienda y el bienestar de los hogares beneficiarios del mismo. Las principales conclusiones del informe del BID fueron: El déficit habitacional está concentrado en el quintil de población más pobre; la focalización es adecuada pero el acceso de la población en déficit es mínimo y la población objetivo cubierta es pequeña; los hogares beneficiarios más pobres pueden experimentar el efecto "pobreza inducida por vivienda"; acceso concentrado en hogares de ingreso medio, que cuentan con acceso a crédito y tienen capacidad de ahorro. La evaluación recomendaba: aumentar el tamaño del programa, mejorando el tamaño de los subsidios para los más pobres (La inversión promedio en subsidios entre 1995-2006 fue 0, $28 \%$ del PIB); revisar las condiciones del crédito hipotecario a todo nivel de ingreso. (p.3)

Dentro de esta perspectiva y como parte de la estrategia para la superación de la pobreza extrema se diseñaron nuevos instrumentos para dar acceso efectivo a vivienda a los hogares beneficiarios. Sobre el tema el DNP (2016) afirma:

La primera herramienta necesaria para el desarrollo del Programa era definir el marco para su ejecución, de manera que el Gobierno Nacional promovió la expedición de la Ley 1537 de 2012, la cual implicó grandes innovaciones para la política de vivienda, así: i) Facultó al Fondo Nacional de Vivienda (Fonvivienda) para constituir patrimonios autónomos que administraran sus recursos con el 
propósito de ejecutar proyectos de vivienda de interés prioritario urbana, ii) Permitió que tanto las entidades públicas como las privadas aportaran recursos a estos patrimonios, y autorizó que las entidades públicas transfirieran a título gratuito lotes de su propiedad a los referidos fideicomisos, iii) Permitió que se transfieran a los patrimonios autónomos los recursos de subsidios familiares de vivienda objeto de renuncia, los vencidos, los recuperados mediante actuaciones administrativas y los que no han sido aplicados. (p.1)

Como resultado, para efectos de la ley se considera que las viviendas de interés social y de interés prioritario (VIS y VIP) son aquellas que se construyen para atender la demanda de los estratos cero a tres. En el caso de las VIS no superan un costo de $\$ 73.671 .000$ $y$ las VIP de $\$ 39.669 .000$. Los instrumentos diseñados para los programas de vivienda de acuerdo a la población objetivo fueron los siguientes:

Para la población vulnerable se presentó el programa vivienda gratuita (ley 1537 de 2012), a los hogares con ingresos entre 1 y 2 salarios mínimos (SMLMV) se dirigió el programa de vivienda para ahorradores, hacia los hogares con ingresos hasta 8 SMLMV se ofreció el programa de subsidio a la tasa de interés para VIP y VIS (FRECH 2) y dirigido a hogares de clase media el programa de subsidio a la tasa de interés para No VIS entre 135 y 335 SMLMV (PIPE).

\section{Efectividad y Procesos en Proyectos Sociales}

\section{En opinión de Fernández (2000)}

La razón última de un proyecto social es solucionar un determinado problema y en este sentido, el concepto de eficacia determina el grado en que se alcanzan los objetivos y metas del proyecto en la población usuaria, en un periodo de tiempo determinado e independientemente de los costes que ello implica. Por otra parte, el concepto de eficiencia, como es sabido, se asocia a la productividad, al relacionar recursos con resultados y traducirlo todo a unidades monetarias. Pero los resultados de un programa deben ser también analizados e interpretados de acuerdo con los procesos desarrollados para su consecución esto es, con su efectividad. (p.260)
Agrega Fernández (2000) "la evaluación de la efectividad o control de procesos puede llegar a explicar la eficacia y la eficiencia de un determinado programa, aspectos que por sí solos proporcionan una imagen incompleta del programa" (p.260). Sabemos que se puede ser muy eficaz, pero sin eficiencia, y también es frecuente ver acciones sociales eficientes, pero ineficaces, especialmente en temas de atención especializada y de apoyo a las personas en situaciones de riesgo. En nuestra opinión debe considerarse también la continuidad y sostenibilidad del programa como un factor crítico de éxito para asegurar que no se enfocará solo en situaciones coyunturales debido a las connotaciones políticas que se le atribuyen a los programas sociales.

\section{Metodología}

El trabajo se desarrolla con un enfoque cuantitativo, se aplicó análisis documental y como técnica la revisión documental, la mayor parte de la información para garantizar su confiabilidad fue obtenida en bases de datos de organismos oficiales vía internet. Se utilizó un diseño no experimental transeccional.

Una vez obtenida la información se clasificó y organizó mediante tablas de registro y se adaptó para el análisis y desarrollo de los objetivos planteados. Para efectuar el análisis matemático y estadístico se utilizó el programa Excel de Microsoft ${ }^{\circledR}$. La técnica aplicada fue el análisis mediante estadística descriptiva. Para caracterizar el mercado inmobiliario en Colombia, se investigó la estructura actual del mercado inmobiliario en Colombia con un enfoque macro-micro (Nacional-Cúcuta). Posteriormente, se analizaron las características y variables de la Ley 1537 de 2012. Este objetivo se desarrolló con una revisión documental. Seguidamente se procedió a analizar la colocación de vivienda y crédito hipotecario en Cúcuta a raíz de la Ley 1537 de 2012; se contrastaron las viviendas construidas por tipo VIP y no VIS y el número y monto de créditos otorgados. Finalmente se analizó la eficacia de la Ley 1537 de 2012 en la adjudicación de créditos hipotecarios en cada uno de los estratos socio económicos en la ciudad de Cúcuta, para ello se efectuó una revisión documental donde se examinó la colocación de créditos hipotecarios y la construcción de viviendas en la ciudad de Cúcuta, esto se hizo por estratos de in- 
gresos. Con la finalidad de conocer posiciones sobre la efectividad del programa se consultó la opinión de Camacol como gremio del sector construcción, la posición de un investigador académico, un estudio de BBVA Research (2015), un artículo de prensa y se incorporaron opiniones de la Contraloría General de la República (2013) al programa de vivienda, así como la posición del Gobierno ante las mismas.

\section{Resultados}

Se evaluó la evolución del déficit de vivienda en Colombia tomando como referencia los censos de población de los años 1997 y 2005 junto con los datos del año 2012 de Fedesarrollo (2012) y BBVA Research (2015) (Ver tabla 1).

Tomando como referencia el censo del año 1993 se evidencia, que el déficit de vivienda en Colombia se ha venido reduciendo. El indicador pasó del 47,3\% en el año 1997 al 12,6\% en el año 2012, entre los años 1993 y 2005 se redujo el $20,30 \%$ representando una variación interanual del $1.7 \%$, mientras que entre los años 2005 y 2012 la reducción fue del $14,40 \%$ equivalente al $2.1 \%$ interanual.

TTabla 1.

Déficit de vivienda años 1993, 2005 y 2012

\begin{tabular}{|c|c|c|c|c|c|}
\hline Año & $\begin{array}{c}\text { Déficit cuantitativo } \\
\text { (unidades) }\end{array}$ & Variación (\%) & $\begin{array}{r}\text { Déficit cualitativo } \\
\text { (unidades) }\end{array}$ & Variación (\%) & $\begin{array}{c}\text { Déficit de vivienda } \\
(\%)\end{array}$ \\
\hline 1993 & 1.093 .077 & & 1.341 .730 & & $47,3 \%$ \\
\hline 2005 & 1.031 .256 & $-5,7 \%$ & 1.185 .607 & $-11,6 \%$ & $27,0 \%$ \\
\hline 2012 & 554.087 & $-46,3 \%$ & 1.093 .006 & $-7,8 \%$ & $12,6 \%$ \\
\hline
\end{tabular}

Fuente: elaboración propia basada en Fedesarrollo $(2012$, p.9) y BBVA Research $(2015$, p.5)

En cuanto al área aprobada para vivienda la información correspondiente entre enero 2011 y enero 2015 para todo el país se muestra en la figura 1.

Se detallan tres comportamientos en el periodo, primero la caída del área aprobada entre el año 2011 y final del año 2012, a partir del año 2012 año de entrada en vigencia de la Ley 1537 se rompe la tendencia a la baja que presentaba el indicador y se inicia un periodo de recuperación que se mantiene hasta finales del año 2014. En 2014, el comportamiento cambió lentamente, pero de cara a la actividad de 2015, dado que el rezago de la cons- trucción respecto a las licencias es aproximadamente de ocho meses. Respecto a la evolución del número de vivienda VIS y no Vis construidas se detalla que la construcción de vivienda VIS amparada por la Ley 1537 supera ampliamente a la vivienda No VIS en dos periodos claramente definidos (Ver figura 2).

Las viviendas destinadas a VIS crecieron aproximadamente $53 \%$ en 2013 , mientras que las dirigidas a vivienda de mayor lo hicieron a una tasa aproximada de $0,10 \%$. En cuanto a las unidades de vivienda iniciadas de acuerdo al rango de precio en la figura 3 se presenta la distribución porcentual. 
-JFigura 1.

Variación del área aprobada para vivienda Enero 2011-enero 2015

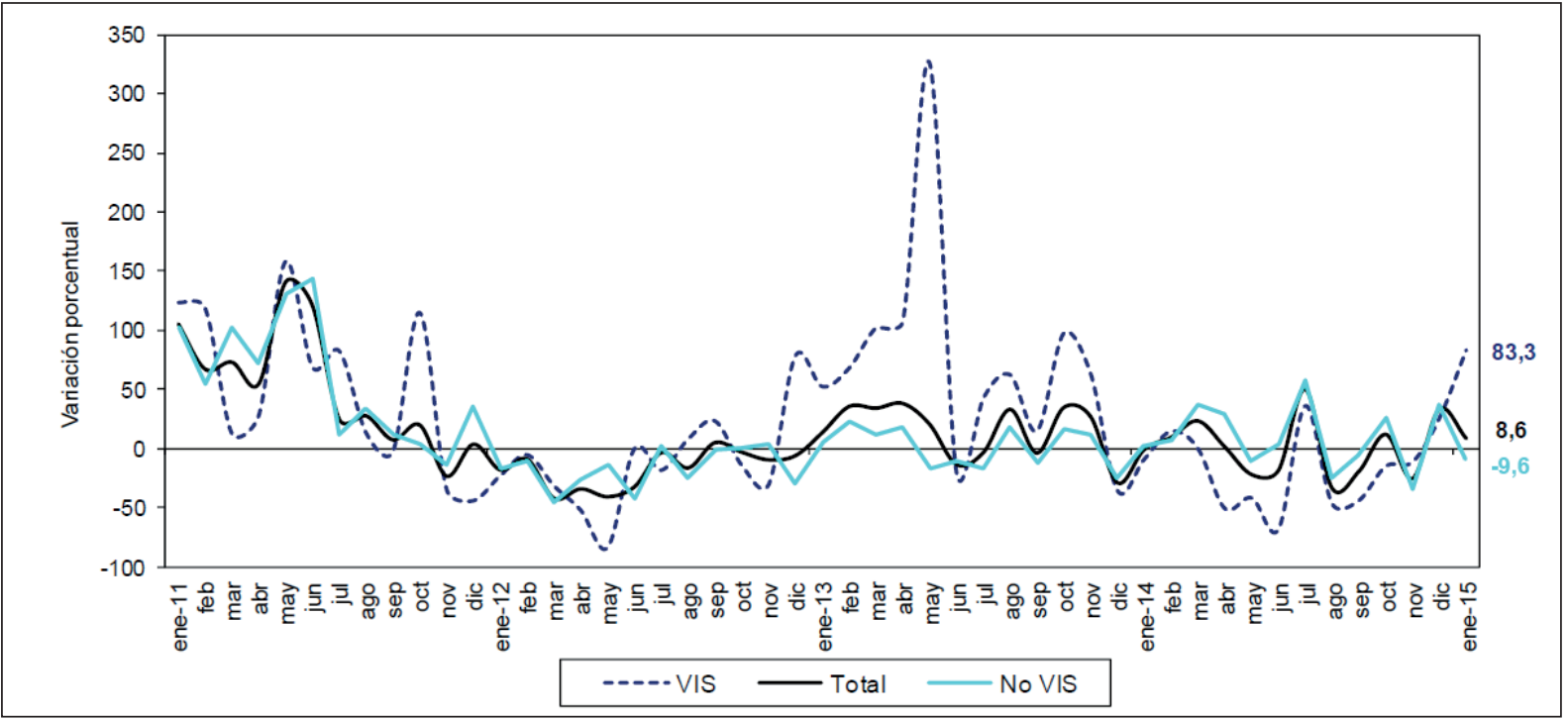

Fuente: DANE (2014) Estadísticas de Edificación Licencias de Construcción

Figura 2.

Variación porcentual porcentaje de viviendas VIS y No VIS construidas

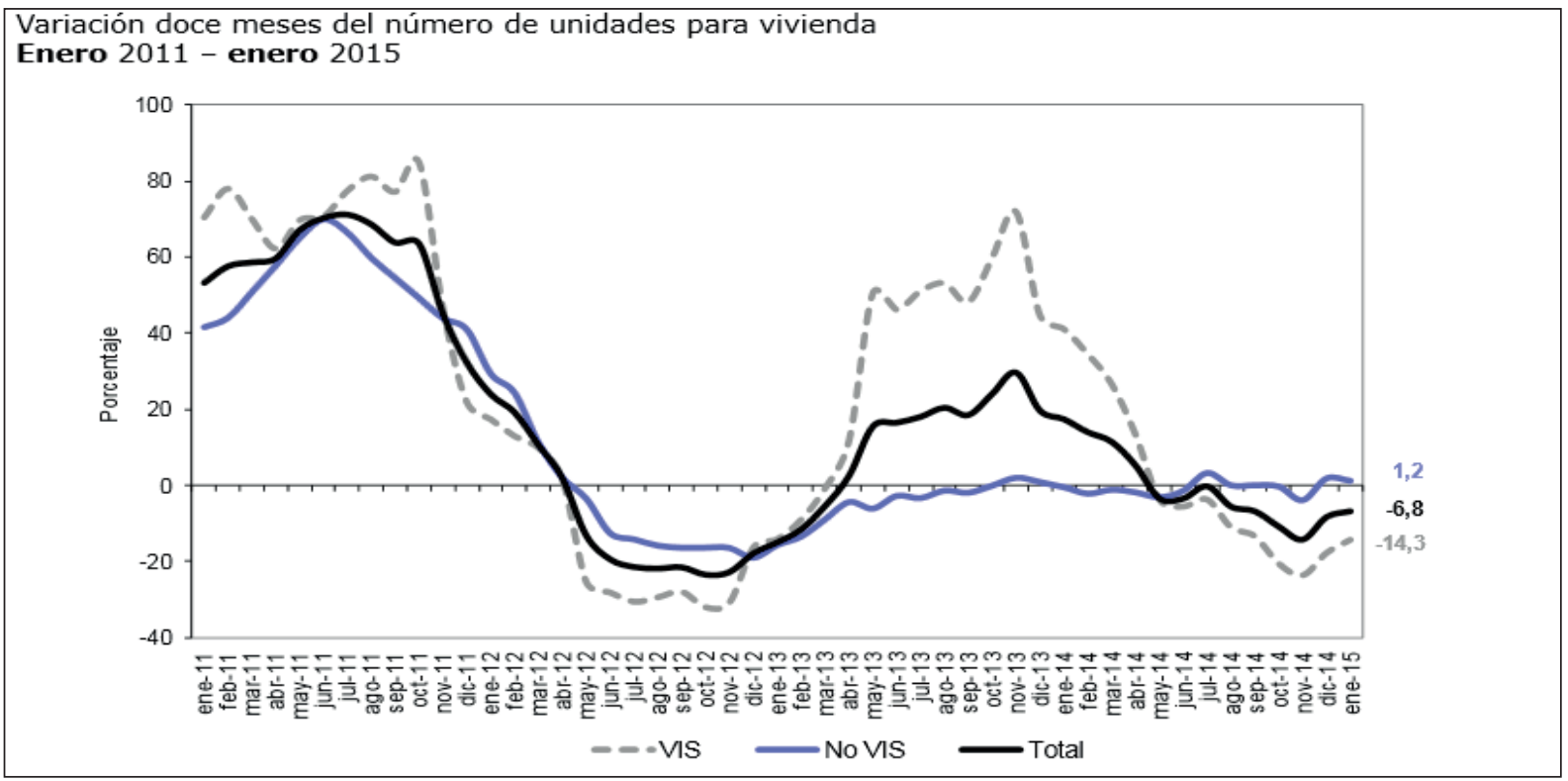

Fuente: DANE (2014) Estadísticas de Edificación Licencias de Construcción 
Figura 3.

\section{Unidades iniciadas de vivienda según rango de precio}

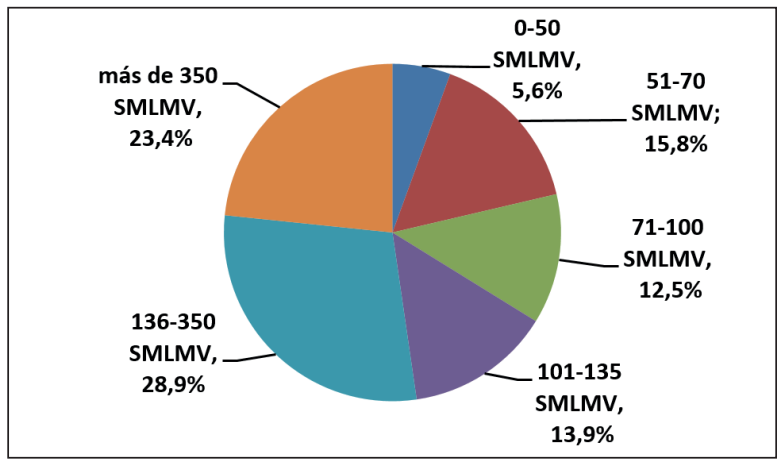

Fuente: elaboración propia basada en DANE (2015) Vivienda VIS y no VIS Series Históricas 15 áreas de influencia III 2007-IV 2015

Se verifica que los estratos 2, 3 y 4 representaron el $42,1 \%$ de las unidades iniciadas, mientras que al estrato 1 correspondió el 5,6\% reflejándose el impacto de las políticas.

En atención a la problemática expuesta se indagó sobre las características y variables de la Ley para la adjudicación de créditos hipotecarios en Colombia. En Fedesarrollo (2012) se dice:

(...) el Plan Nacional de Desarrollo (PND) identificó como principales restricciones al crecimiento del sector la baja disponibilidad de suelo para VIS, la limitación en el acceso a la financiación y la baja articulación entre el Subsidio Familiar de Vivienda (SFV), el otorgamiento de crédito y la generación de oferta. (p.9)

En la búsqueda de evidencias en Camacol (2012) dice "el modelo de cierre financiero, sobre el cual se erige la política de vivienda, se sustenta en la premisa de que cada comprador debe gestionar por su cuenta tres fuentes de recursos para la compra de una vivienda" (p.3).

Se plantean entonces en Camacol (2012):

(...) en buena parte de los hogares que potencialmente son objeto de la política de vivienda, es decir, aquellos hogares con ingresos infe- riores a 4 SMMLV, no están pudiendo lograr el cierre financiero. En ese orden de ideas, la población objetivo de la política de vivienda se puede dividir en dos grandes grupos: los hogares con acceso a crédito y con posibilidades de ahorro y los hogares sin posibilidad de cierre financiero. (p.4)

Indagando sobre el porcentaje de hogares con déficit de vivienda en el Departamento de Norte de Santander, en la figura 4 se presenta dicha información según los censos de los años 1993 y 2005.

Figura 4.

\section{Porcentaje de hogares en déficit de vivienda Cúcuta y Área metropolitana (A.M)}

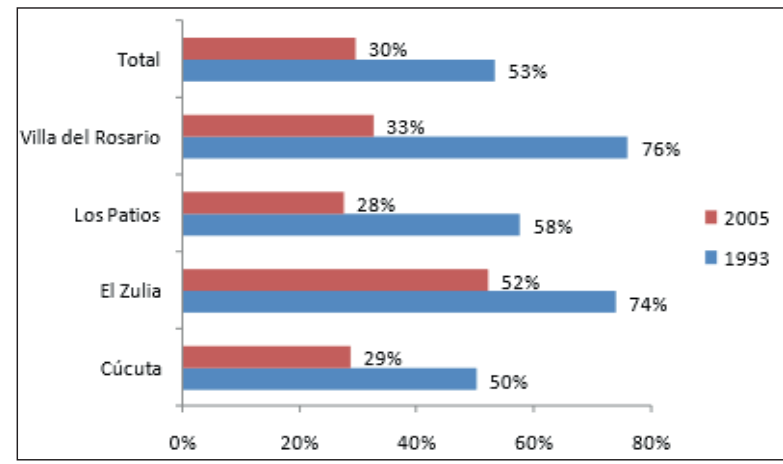

Fuente: Camacol (2011). Estudio de demanda de vivienda en Cúcuta y Área metropolitana

Se determinó que en el Departamento de Norte de Santander hubo una disminución del $23 \%$ en el déficit de hogares entre los años 1993 y 2005. De hecho, en la ciudad de Cúcuta la reducción fue del $21 \%$ en ese periodo. Ahora bien, en cuanto a la producción de vivienda VIS y no VIS en la ciudad de Cúcuta se muestra el comportamiento en la figura 5.

Es evidente que en la ciudad de Cúcuta a partir del año 2012 la producción de viviendas VIS y no VIS repuntó significativamente. La producción de vivienda VIS se incrementó 317,8\% en el año 2013 con respecto al año 2012 y el 64,4\% en el año 2014 con respecto al año 2013, mientras que la vivienda no VIS aumento el 266,5\% en el año 2013 y se contrajo $39,7 \%$ entre los años 2013 y 2014. 
Complementando el análisis se analizó el comportamiento de la cartera hipotecaria, se encontró que el número de créditos hipotecarios que apoyaron la construcción tuvo un incremento sostenido que represento el 19,6\% de aumento en la cartera colocada tal como se observa en la figura 6 .

Figura 5.

Producción de VIS y No VIS en Cúcuta, años 2007 - 2014 (III Trimestre)

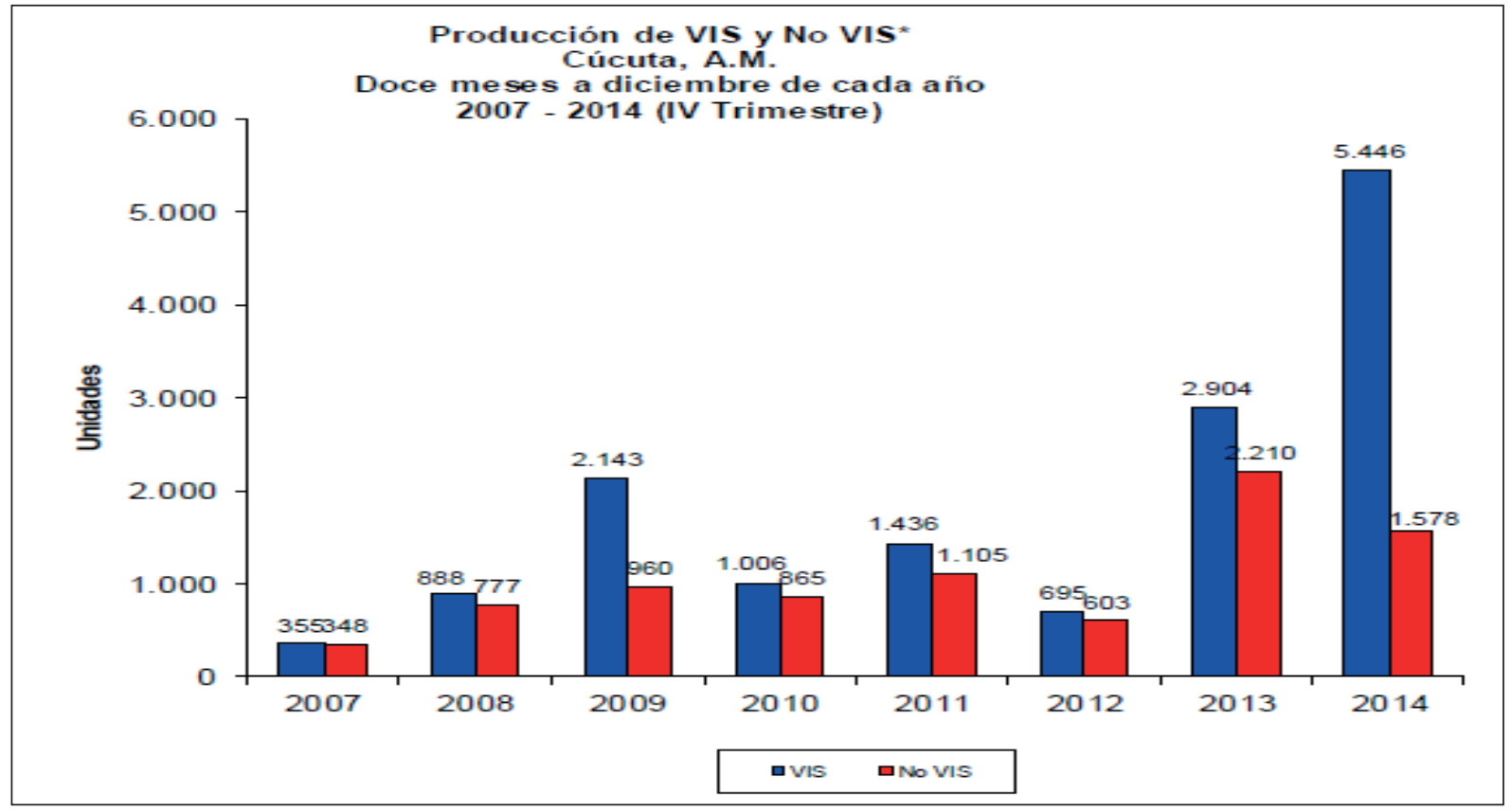

Fuente: CENAC (2015) Boletín estadístico - contexto sectorial Cúcuta - Norte de Santander

IFigura 6.

Número de créditos hipotecarios en Norte de Santander

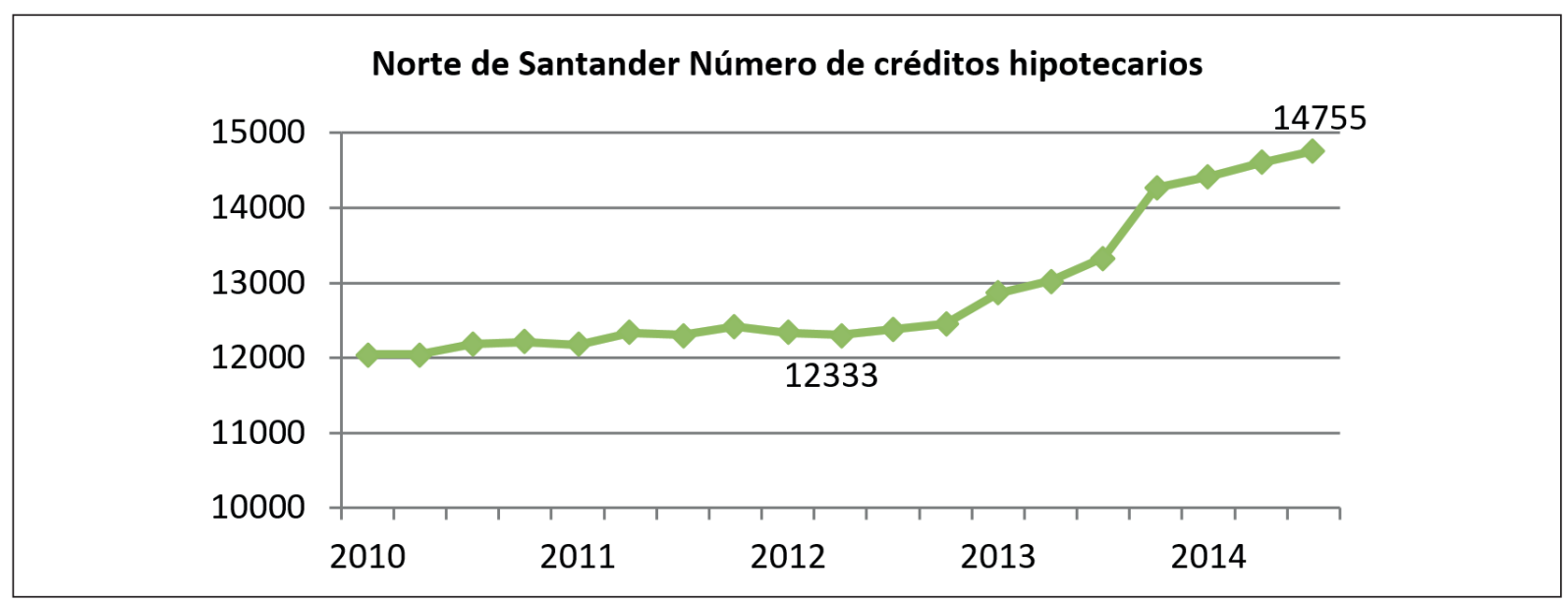

Fuente: elaboración propia basada en CENAC (2015) 
En la tabla 2 se detalla el valor de los créditos entregados para la compra de vivienda desde el ámbito nacional al local entre los años 2010-2014, comparando la colocación de cartera entre el sistema bancario y el resto del sistema.

Se detalla que entre los años 2010-2014 la participación del sistema bancario en la colocación de cartera hipotecaria en la ciudad Cúcuta A.M. estuvo por encima del resto del sistema. El sistema bancario presentó un crecimiento sostenido en el porcentaje de financiación al pasar de 56,1\% en el año 2010 a 81\% en el año 2014 mientras en el resto del sistema se evidenció una caída de 20,3\% en el año 2013 con respecto al año 2012. Con las evidencias anteriores se resume el comportamiento del sector vivienda en Cúcuta en los años posteriores a la vigencia de la Ley 1537 de 2012 (Ver tabla 3).

Tabla 2.

Colocación de cartera hipotecaria en Cúcuta (Millones de Pesos)

\begin{tabular}{|l|c|c|c|c|c|}
\hline & 2010 & 2011 & 2012 & 2013 & 2014 \\
\hline Cartera Hipotecaria & $146.423,00$ & $173.948,00$ & $200.242,00$ & $119.099,00$ & $121.590,00$ \\
\hline variación \% & - & $18,8 \%$ & $15,1 \%$ & $-40,5 \%$ & $2,1 \%$ \\
\hline \% sistema bancario & $56,1 \%$ & $56,4 \%$ & $59,1 \%$ & $79,4 \%$ & $81,0 \%$ \\
\hline \% resto del sistema & $43,9 \%$ & $43,6 \%$ & $40,9 \%$ & $20,6 \%$ & $19,0 \%$ \\
\hline
\end{tabular}

Fuente: elaboración propia basada en DANE (2013) Informe de Coyuntura Económica Regional y Datacucuta (2011)

Tabla 3.

\section{Comportamiento del sector construcción de vivienda en Cúcuta 2013-2014}

\begin{tabular}{|c|c|c|c|c|c|}
\hline Año & $\begin{array}{l}\text { Oferta total } \\
\text { en unidades } \\
\text { (incluye } \\
\text { lanzamiento } \\
\text { de proyectos, } \\
\text { iniciaciones, y } \\
\text { ventas) }\end{array}$ & $\begin{array}{l}\text { Metros cuadrados } \\
\text { licenciados para } \\
\text { la construcción }\end{array}$ & $\begin{array}{c}\text { Licencias } \\
\text { otorgadas para } \\
\text { la construcción } \\
\text { de residencia } \\
\text { (metros } \\
\text { cuadrados) }\end{array}$ & $\begin{array}{l}\text { Ventas de } \\
\text { viviendas } \\
\text { (unidades) }\end{array}$ & $\begin{array}{l}\text { Lanzamiento } \\
\text { de proyectos } \\
\text { de viviendas } \\
\text { (unidades) }\end{array}$ \\
\hline 2013 & 29763 & 321674 & 267620 & 2808 & 2360 \\
\hline 2014 & 42840 & 306356 & 243030 & 6244 & 6656 \\
\hline
\end{tabular}

Fuente: elaboración propia basada en Camacol (2015) Estudio de demanda de vivienda en Cúcuta y su área metropolitana

En la oferta total se observó una variación del 43,94\% entre los años 2013 y 2014, mientras que en los metros cuadrados licenciados para la construcción se presentó una desaceleración del 5\%, por otra parte, en las licencias otorgadas para la construcción la reducción fue del $9 \%$. Se encontró también que las ventas de viviendas (unidades) se incrementaron el $122 \%$ y de igual manera el lanzamiento de proyectos de viviendas (unidades) creció el $282,03 \%$. En la tabla 4 se presenta el comportamiento de las ventas de vivienda VIS correspondiente a los estratos 1 y 2 . 
Tabla 4.

\section{Venta de viviendas VIS estratos 1 y 2}

\begin{tabular}{|c|c|c|c|c|}
\hline & $\begin{array}{c}\text { Ventas VIS } \\
\text { Estratos 1 y 2 }\end{array}$ & Ventas No VIS & \% Multifamiliares & \% Unifamiliares \\
\hline 2013 & 1434 & 1278 & 70 & 30 \\
\hline 2014 & 3889 & 2278 & 75 & 25 \\
\hline Variación \% & $+171 \%$ & $+78 \%$ & $+7 \%$ & $-17 \%$ \\
\hline
\end{tabular}

Fuente: elaboración propia basada en Camacol (2015) Estudio de demanda de vivienda en Cúcuta y su área metropolitana

Se encontró que las ventas VIS en los estratos 1 y 2 aumentaron el $171 \%$ mientras que las ventas no VIS crecieron el $78 \%$ junto al aumento de la construcción de sistemas multifamiliares y caída en los sistemas unifamiliares. Según estos resultados se pudiera inferir que son consecuencia de las facilidades otorgadas por los programas de vivienda.

De modo ilustrativo en la tabla 5 se muestra como se distribuyeron las unidades vendidas por estrato en Cúcuta en el año 2014.

\section{Tabla 5.}

Unidades vendidas por estrato en Cúcuta en el año 2014

\begin{tabular}{|c|c|c|}
\hline & Unidades & $\%$ \\
\hline Estrato 1 & 185 & 6 \\
\hline Estrato 2 & 464 & 15 \\
\hline Estrato 3 & 1854 & 60 \\
\hline Estrato 4 & 516 & 17 \\
\hline Estrato 5 & 44 & 1 \\
\hline Estrato 6 & 24 & 1 \\
\hline
\end{tabular}

Fuente: elaboración propia basada en Camacol Cúcuta \& NorOriente (2015)

El estrato socioeconómico con mayor participación en la compra de vivienda fue el número tres con el $60 \%$ de las unidades vendidas, le siguió el estrato número cuatro con el $17 \%$, al estrato 2 le correspondió el $15 \%$ y el estrato uno con el $6 \%$. El menor porcentaje le correspondió a los estratos cinco y el seis con el $1 \%$ a cada uno. A continuación, en la tabla 6 se muestra el número de viviendas financiadas en los años 2012 y 2013 en el Norte de Santander-Cúcuta.

El porcentaje de viviendas nuevas financiadas representó el 41,3\% en 2012 y se incrementó al 52,6\% en 2013 mientras que en Cúcuta este mismo sector pasó del 36,7\% en el 2012 al 53,1\% en el 2013. Por su parte el mercado de viviendas usadas pasó de $63,3 \%$ en 2012 a 46,9\% en 2013. Resulta claro el impacto positivo en el sector vivienda en la región.

Como seguimiento al cumplimiento de la política de vivienda se presentan a continuación diferentes opiniones desde diversas perspectivas:

En Camacol (2012) se encontró:

(...) el nuevo esquema planteado en la ley 1537 de 2012 es un paso en la dirección correcta. La construcción de las primeras 100.000 viviendas de interés prioritario se constituyen como un primer paso para llenar el vacío existente en torno a los mecanismos de acceso a vivienda disponibles para los hogares que no pueden lograr su adquisición a través del modelo de cierre financiero, además de ser un complemento necesario a la meta del millón de viviendas. (Camacol, 2012). Agregan finalmente que la construcción de VIP se robustece en los departamentos que cuentan con una oferta importante de recursos que incentiven la demanda. (p.1) 
Número de viviendas financiadas 2012 - 2013 Nacional - Norte de Santander - Cúcuta.

\begin{tabular}{|c|c|c|c|c|c|c|}
\hline \multirow{2}{*}{ Región } & \multicolumn{2}{|c|}{ Nueva } & \multirow{2}{*}{ Variación \% } & \multicolumn{2}{|c|}{ Usada } & \multirow{2}{*}{ Variación \% } \\
\hline & 2012 & 2013 & & 2012 & 2013 & \\
\hline Nacional & 66.764 & 82.466 & 23,5 & 55.290 & 60.000 & 8,5 \\
\hline Norte de Santander & 810 & 1.356 & 67,4 & 1.149 & 1.220 & 6,2 \\
\hline Cúcuta & 550 & 1.087 & 97,6 & 950 & 962 & 1,3 \\
\hline
\end{tabular}

Fuente: DANE (2013) ICER Norte de Santander

Por su parte en un estudio del BBVA Research (2015) se señala:

(...) las medidas de asequibilidad de la vivienda (número de años que tarda una familia promedio colombiana, destinando todos sus ingresos después de impuestos para vivienda (sin hacer gastos adicionales en alimentos, viajes) para comprar una casa de precio medio en el país) muestran que por primera vez desde 2010 no se dieron aumentos en el número de años necesarios para comprar vivienda. (...) se destacó una reducción marginal de la tasa de interés para viviendas no VIS, desde 10,8\% hasta 10,7\%. También, la tasa VIS sigue siendo una de las más bajas de la historia al ubicarse en $11,6 \%$. Exponen que el crédito hipotecario sigue ganando participación en la economía y ahora se ubica en el $6,3 \%$ del PIB esa misma medida era de $5,9 \%$ al cierre de 2013. (pp.1, 2)

Ahora bien, Mesa (2015) opina:

(...) el programa ha tenido un efecto positivo sobre la demanda de crédito. Sin embargo, con información hasta 2014, observa que la evolución de los nuevos créditos totales de vivienda ha seguido los ciclos del programa, creciendo de manera significativa en los meses inmediatamente siguientes a los anuncios del gobierno sobre los recursos y cupos del subsidio en cada nueva etapa y cayendo a medida que éstos se van agotando. Ello sugiere que el efecto del programa sobre la profundización del crédito hipotecario es más bien transitorio y limitado. Para Mesa esto no sorprende, pues depende en última instancia del monto de recursos públicos que el Presupuesto puede apropiar para el Fondo de Rescate de Crédito Hipotecario (FRECH), lo que a su vez determina el número de subsidios que es posible extender en cada uno de los segmentos objetivo. (p.10)

Por su parte la Contraloría General de la República (CGR) (2013) manifiesta:

(...) para 2012 el Fondo Nacional del Ahorro (FNA) proyectó aprobar 85.500 créditos hipotecarios por valor de $\$ 2,82$ billones de Pesos de los cuales sólo se aprobó el 63,5\%, equivalente a 54.339 créditos (por un valor total de $\$ 2,56$ billones, correspondiente al $90,6 \%$ del valor proyectado). Complementa indicando que el FNA proyectó desembolsar 37.950 créditos hipotecarios (que corresponden al $44,4 \%$ de las aprobaciones proyectadas), de los cuales solo se desembolsaron efectivamente 20.717 por un monto de $\$ 928.212$ millones, que corresponden al $54,6 \%$ de los desembolsos proyectados y apenas al 38,1\% de los créditos aprobados. (...) con lo anterior se reafirma la existencia de un importante rezago entre los créditos aprobados y los efectivamente desembolsados por el fondo, situación preocupante porque indica que gran parte del esfuerzo realizado por el Estado a través del FNA para facilitar el acceso de los colombianos a la vivienda no se está materializando en la adquisición de viviendas por parte de las familias. (p.7)

Ante el informe de la CGR en "Minvivienda responde a Contraloría por casas gratis" (7 sep. 2015) en 
Semana.com, el Ministro de Vivienda manifestó estar en desacuerdo con las cifras presentadas y solicitó una revisión conjunta de las mismas. Con respecto a esta información no se obtuvo más detalle.

Se consultó en la prensa y se encontró en el diario El Tiempo en "Locomotoras, en recta final del cuatrienio" (2014, 18 de enero) el siguiente comentario:

(...) sumar un millón de viviendas durante el cuatrienio parece posible. Van 675.000 y se destacan las 100.000 unidades del programa de gratuidad, de las cuales el 2013 cerró con 40.000 terminadas. Según el avance de objetivos del Plan de Desarrollo, el número de asignaciones va en el 14 por ciento.

Como se puede observar las opiniones sobre el cumplimiento de la meta del programa de vivienda son encontradas, no obstante, se evidencia un repunte en la construcción, financiación y venta de vivienda en la ciudad de Cúcuta después de la entrada en vigencia de los programas de vivienda y la Ley de Vivienda 1537 de 2012.

\section{Conclusión}

El análisis precedente permite concluir que con la aprobación de la Ley de Vivienda se dotó al país de una herramienta que coadyuva en la atención de un sector de la población que por su condición de pobreza no tiene posibilidades de acceder a una vivienda digna, así como atacar el déficit habitacional. Con esto se apoya el derecho fundamental consagrado en la Constitución Nacional como es el tener acceso a una vivienda. Desde la perspectiva más general es importante hacer notar que a partir del año 2012 se rompe la tendencia a la baja en cuanto al área aprobada para vivienda y se inicia un periodo de recuperación que se mantiene hasta finales del año 2014 cuando se desacelera el ritmo de aprobación lo cual puede ser debido al desfase con el proceso de construcción y colocación.

Por lo demás la construcción de vivienda VIS supera ampliamente a la vivienda No VIS. Se espera un impulso a la vivienda social con el programa Mi Casa Ya a partir de enero de 2015 y hasta 2018. Es conveniente anotar que uno de los problemas que enfrenta el programa VIP radica en las dificultades y desconocimiento respecto al cierre financiero en que están incurriendo los hogares eventualmente beneficiarios de ese programa. Sobre ese tema el Estado debe profundizar su apoyo apuntalando y educando financieramente a ese sector más pobre.

En el marco de la investigación es de hacer notar que a partir del año 2012 la producción de viviendas VIS y no VIS repuntó significativamente y se apreció el crecimiento sostenido en la colocación de cartera hipotecaria en el Departamento de Norte de Santander. Posterior a entrada de la Ley 1537 de 2012 el sector se dinamizó en su conjunto dado que los programas de vivienda que se implementaron por el gobierno nacional impactaron positivamente el sector.

Aunque las opiniones son encontradas sobre el cumplimiento y la efectividad de este programa es evidente que el programa presentó un balance positivo en la ciudad de Cúcuta una vez en vigencia la Ley 1537 de 2012. No obstante, debe profundizarse en aspectos de apoyo logístico a los diferentes programas de vivienda. Se plantea entonces tomar medidas entre el municipio y el Ministerio de Vivienda, Ciudad y Territorio que permitan consolidar los programas de vivienda VIP y VIS. Esas medidas podrían incluir el aumento de la inversión en vivienda y subsidios, igualmente ubicar y proponer el suelo urbano con factibilidad y disponibilidad para los servicios. Se recomienda al Estado y a la Banca profundizar en el proceso de cierre financiero para apoyar a los hogares que presentan problemas en esa fase del proceso; se requiere profundizar en la educación financiera para apoyar a ese segmento de la población.

Se debe profundizar en la coordinación y asociaciones en los macro proyectos, planes y coordinación de políticas de vivienda entre la nación, las gobernaciones, las áreas metropolitanas, los municipios, el sector financiero, académico y el sector privado. Aunque ya existen coordinaciones por proyectos la propuesta es convertirlas en política de Estado permanente que no solo atienda situaciones puntuales. Es un hecho que la implementación del programa conlleva una serie de beneficios para los hogares que reciben una solución habitacional, para el Estado al cumplir con uno de sus roles fundamentales de coadyuvar en la mejora de la calidad de vida de 
La política de vivienda y la adjudicación de créditos hipotecarios en la ciudad de Cúcuta

los habitantes y para la sociedad en su conjunto al reivindicar el derecho a vivir en seguridad, paz y dignidad en alguna parte. Importa y por muchas razones apoyar el programa de vivienda debido a que el bienestar de las familias se incrementa por la mejora en las condiciones habitacionales y del entorno; el acceso a servicios públicos básicos; mayor percepción de seguridad. El programa contribuye a disminuir el desempleo dado que utiliza intensivamente personal poco calificado $y$, por ende, de baja remuneración.

Dentro de esta perspectiva debe asegurarse un impacto ambiental claramente positivo, las condiciones de habitabilidad deberán ser mejoradas con relación al asentamiento informal. Igualmente se debe minimizar el riesgo de afectación por desastres naturales, y considerar la dotación de áreas verdes y servicios comunitarios.

\section{Referencias Bibliográficas}

Alcalá, L.C. (2005). La crisis de la vivienda. En Documentación Social 138 pp.81-100. Recuperado de http://www.caritas.es/imagesrepository/ CapitulosPublicaciones/508/05-\%20LA\%20CRISIS\%20 DE\%20LA\%20VIVIENDA.pdf

BBVA Research. (2015). Situación Inmobiliaria Colombia Año 2015. Recuperado de https://www.bbvaresearch.com/wp-content/uploads/2015/03/SituacionInmobiliariaPublicar1.pdf

Camacol (2012). Subsidios plenos y la nueva ley de vivienda y desarrollo urbano. Limitaciones del modelo de cierre financiero frente al acceso a la VIP. Informe Económico No.38, Junio de 2012 .Recuperado de: http://camacol. co/sites/default/files/secciones_internas/Informe $\% 20$ Econ\%C3\%B3mico-Julio-No\%2038_0.pdf

Camacol. (2015). Estudio de demanda de vivienda en Cúcuta y su área metropolitana años 2011-2012-2013 y 2014. Recuperado de: http://www.camacolcucuta.co/style/ documentos/estudios/Estudio-Demanda-Vivienda-2015.pdf

Camacol Cúcuta \& NorOriente. (2015). Informe de Ventas Enero- Septiembre 2015. Coordenada Urbana Camacol. Recuperado de: http://camacolcucuta.co/ style/documentos/2/informe-2015/INFORME-ENEROSEPTIEMBRE-2015-COORDENADA-URBANA.pdf

Cárdenas, M., \& Hernández, M. (2006). El Sector Financiero y la Vivienda. Estudios Fedesarrollo 2006. Recuperado de http://www.repository.fedesarrollo.org.co/
bitstream/11445/984/1/Repor_Marzo_2006_Cardenas_y_ Hernandez.pdf

Carrión, F. (2005). "Vivienda y Crisis". Opinión personal. Recuperado de http://works.bepress.com/ fernando_carrion/201

CENAC (2015). Boletín estadístico - contexto sectorial Cúcuta - Norte de Santander. Actualizado a: Enero de 2015. Recuperado de http://www.CENAC.org. co/?apc=I1----\&x=20152630

Comité de derechos económicos, sociales y culturales (CESCR) de Naciones Unidas. Recuperado de http://www.derechoshumanos.net/ONU/ComiteDerechosEconomicosSocialesCul turales-CESCR.htm

Contraloría General de la República. (2013). Especial Seguimiento a los recursos de Vivienda Urbana. Fondo Nacional del Ahorro y sus recursos. Informe $N^{\circ}$ 20. Julio 2013. Recuperado de: http://www.contraloria.gov.co/documents/155638087/174895202/ Informe_vivienda_20.pdf/7265a931-18b4-42da-9197$6 e 286 \mathrm{~d} 17 \mathrm{c} 31 \mathrm{~b}$ ?version $=1.0$

DANE. (2013). Informe de Coyuntura Económica Regional. Recuperado de http://www.dane.gov.co/index.php/estadisticas-regionales/ informe-de-coyuntura-economica-regional-icer

DANE. (2014). Boletín técnico -Variación doce meses del área total aprobada para vivienda y tipo de vivienda. Recuperado de: https://www.dane.gov.co/files/investigaciones/boletines/licencias/bol_lic_ene15.pdf

DANE. (2015). Históricos. Dieciséis áreas. Series históricas 16 área de influencia (III trimestre 2012 - IV trimestre 2014). Recuperado de http://www.DANE.gov.co/index. php/construccion-alias/vivienda-de-interes-social-vis

Datacucuta. (2011). Indicadores económicos de Norte de Santander. Cámara de Comercio de Cúcuta. Recuperado de http://www.datacucuta.com/\#!estudios/cur7

Declaración Universal de Derechos Humanos. Recuperado de http://www.un.org/es/documents/udhr/

Departamento Nacional de Planeación. (DNP). (2016). Subsidio familiar de vivienda para población desplazada-región nacional. Recuperado de: https:// spi.dnp.gov.co/App_Themes/SeguimientoProyectos/ ResumenEjecutivo/1150021230000.pdf

El déficit de vivienda bajó en Colombia. (2014, 13 de Abril). Portafolio. Recuperado de http://www.portafolio.co/ economia/deficit-vivienda-colombia

Fedesarrollo. (2012).¿Cómo va la locomotora de la Vivienda? Tendencia económica. Informe Mensual de Fedesarrollo 120, abril de 2012. Recuperado de http://www.repository. fedesarrollo.org.co/bitstream/11445/2932/1/TE_No_120_ Abril_2012.pdf 
Fernández, S. (2000). La efectividad de los programas sociales. Enfoques y técnicas de la Evaluación de Procesos. Revista de Psicología del Trabajo y de las Organizaciones, Vol. 16, núm. 3, 2000, pp. 259-276. Recuperado de http://www. redalyc.org/pdf/2313/231317662002.pdf

Golay, C \& Özden M. (2007). El derecho a la vivienda. Programa Derechos Humanos del Centro Europa. Tercer Mundo (CETIM). Recuperado de http://www.cetim.ch/wp-content/ uploads/Derecho-a-la-vivienda.pdf

Henao, L.F. (2013). La transformación de la vivienda en Colombia. Definición de una política integral para cada segmento poblacional. Ponencia presentada en la XLVIII Convención Bancaria Asobancaria 2013. Cartagena de Indias, Junio 7 de 2013. Recuperado en http://www.minvivienda. gov.co/Discursos/XLVIII\%20Convenci\%C3\%B3n\%20bancaria\%20ASOBANCARIA\%202013.pdf

Locomotoras, en recta final del cuatrienio. (2014, 18 de enero). El Tiempo. Recuperado de: http://www.eltiempo.com/ archivo/documento/CMS-13380638

Mejía, Cecilia. (Comp.). (2005). Actualidad: el crédito de vivienda: ¿estímulo para la construcción? En Tendencia
Económica. Informe Mensual de Fedesarrollo 45. Diciembre de 2005. Recuperado en: http://www.repository. fedesarrollo.org.co/bitstream/11445/753/1/TE_No_45 Diciembre_2005.pdf

Mesa, C.A. (2015). El bajo nivel de profundización del crédito de vivienda en Colombia. Tendencia Económica. Informe Mensual de Fedesarrollo No. 152 - Febrero 2015. Recuperado de: http://www.fedesarrollo.org.co/wpcontent/uploads/T-E_No._152.pdf

Rodríguez, Raquel. (2010). La política de vivienda en España en el contexto europeo. Deudas y Retos. Revista INVI, [S.L.], V. 25, n. 69, sep. 2010. Recuperado de: http://www.revistas. uchile.cl/index.php/INVI/article/view/8360/8137.

Tella, G. (sf). "La función social del suelo vs el derecho de propiedad". Recuperado en: http://www.guillermotella.com/articulos/ la-funcion-social-del-suelo-vs-el-derecho-de-propiedad/

Zapata, I. (2014). Exclusión Social en el Cinturón Suburbano del Área Metropolitana del Gran Santiago. Urbana, 8(32), 13. Recuperado en: http://saber.ucv.ve/ojs/index.php/ rev_urb/article/view/5997 